\title{
Taponamiento cardíaco debido a perforación de la aurícula derecha por catéter venoso central
}

\author{
Atrium perforation by central venous catheter and cardiac \\ tamponade
}

\begin{abstract}
Resumen
El taponamiento cardíaco es una complicación devastadora de los catéteres venosos centrales. Se debe en general a que la punta del catéter alcanza indebidamente las cavidades cardíacas derechas. Para la correcta identificación en la autopsia por el patólogo forense es importante que a los pacientes fallecidos en los hospitales que van a ser sometidos a autopsia no se les retiren los dispositivos que tengan insertados, e igualmente que no se les retiren en la sala de autopsias hasta que no se haya comprobado su correcta inserción. Se presenta un caso estudiado por nosotros y se analizan otros factores de riesgo de dicha complicación.
\end{abstract}

Palabras clave: Taponamiento cardíaco. Catéter venoso central. Perforación auricular.

\begin{abstract}
Cardiac tamponade is a devastating complication of central venous catheters. It is due to perforation of the atrial or ventricular wall during or after insertion. In order to identify this complication it is important that patients deceased in Hospitals and referred to Forensic Pathologists be sent with all the catheters and devices inserted. Also they should not be removed in the autopsy ward until their correct position has been addressed. A dramatic case is presented and the risk factors for these complications are analyzed.
\end{abstract}

Key words: Heart tamponade. Central venous catheter. Atrium perforation.

\section{Introducción}

El taponamiento cardíaco es una complicación infrecuente, pero devastadora, de los catéteres venosos centrales ${ }^{1}$. Se debe a que la punta del catéter queda indebidamente en el interior de la aurícula o del ventrículo derechos y perfora la pared cardíaca. Todos los casos de muerte por esta causa deberían ser objeto de autopsia médico-legal, tanto si se presentan como una supuesta mala praxis profesional médica o como una muerte inesperada en un paciente hospitalizado². Para el patólogo forense que se enfrenta a uno de estos casos es fundamental el adecuado reconocimiento y la objetivación de dicha complicación en la autopsia.

Hay dos medidas muy importantes en la autopsia médico-legal que venimos practicando desde la puesta en funcionamiento del Servicio de Patología Forense del Instituto de Medicina Legal. La primera es comunicar insistentemente a los hospitales de la provincia que cuando fallezca un paciente que ha de ser sometido a autopsia médico-legal no se le retiren los dispositivos que tenga insertados en el cuerpo (tubos orotraqueales, sondas de todo tipo, drenajes, catéteres intravenosos, etc.); esta petición se hace también a los servicios médicos de emergencias para el caso de pacientes sometidos a medidas de reanimación avanzada y que fallezcan antes de la hospitalización. La segunda medida es que, una vez que el cadáver se encuentre en la sala de autopsias, no se le retiren estos dispositivos hasta finalizada la autopsia o al menos hasta comprobar su correcta colocación u objetivar y documentar los casos en que la situación fuese incorrecta o estos hubiesen causado daños.

Estas medidas nos han permitido la adecuada interpretación de lesiones en el esqueleto gloso-laríngeo, debidas a intubación, en cadáveres de personas que

\section{Ramos Medina ${ }^{1}$ \\ I. Palomo Gómez ${ }^{1}$ \\ PJ. Aranda Granados ${ }^{2}$ \\ JL. Palomo Rando ${ }^{1}$ \\ M. Salguero Villadiego ${ }^{3}$}

${ }^{1}$ Médico Forense. Instituto de Medicina Legal. Málaga ${ }^{2}$ Cirujano Cardiovascular. Hospital Regional Carlos Haya. Málaga ${ }^{3}$ Facultativo del Servicio de Histopatología. Instituto Nacional de Toxicología y Ciencias Forenses. Sevilla

Correspondencia: Valentín Ramos Medina Instituto de Medicina Legal Ciudad de la Justicia Fiscal Luis Portero, 6 29010 Málaga E-mail: valentin.ramos.ius@ juntadeandalucia.es

Fecha de recepción: 22. OCT. 2014

Fecha de aceptación: 24. NOV. 2014 
Figura 1.

Drum antecubital insertado en el antebrazo derecho.

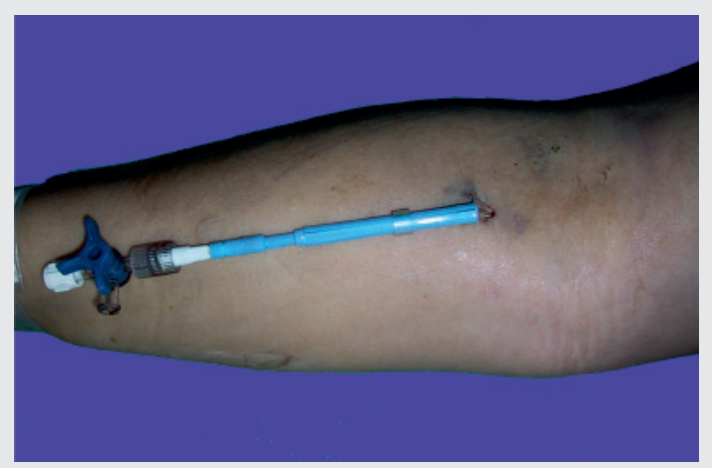

Figura 2

Saco pericárdico intensamente distendido por hemopericardio a tensión.

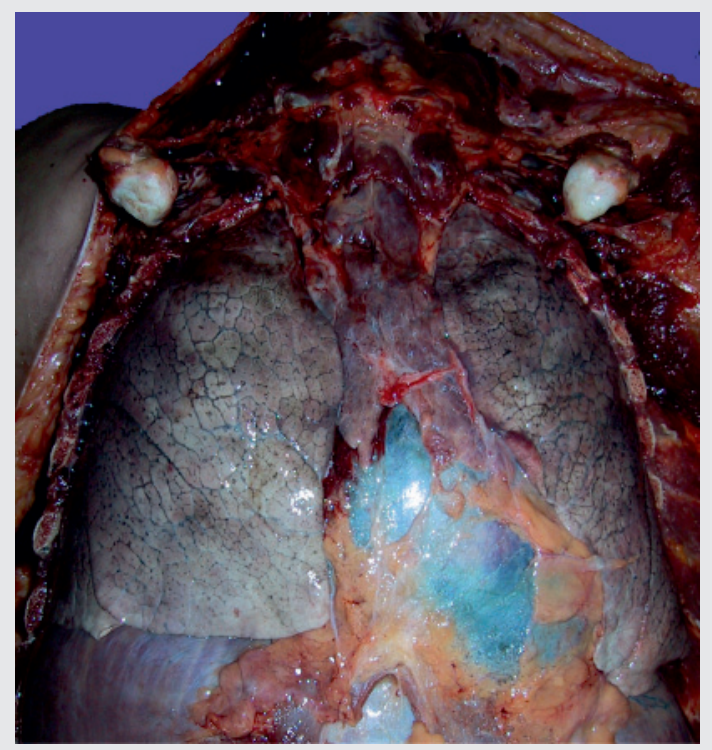

habían sido encontradas inconscientes en la vía pública y que de otra forma hubiesen planteado dudas sobre si podían deberse a intentos de estrangulación u otras agresiones sobre el cuello; igualmente, casos de intubación esofágica o taponamientos cardíacos por catéteres vasculares, como el que comunicamos en este trabajo, cuyo mecanismo de producción hubiera podido plantear dificultades si los catéteres hubiesen sido retirados tras el fallecimiento en el hospital o al iniciar la autopsia.

\section{Presentación del caso}

Varón de 42 años de edad con antecedentes de asma bronquial y varios ingresos hospitalarios por descompensación grave del asma, en concreto en cinco ocasiones durante los 8 años anteriores. En esta ocasión ingresa procedente de su domicilio por sospecha de hemoptisis, y se le practican broncoscopia y esofagogastroscopia que fueron normales. En la mañana del tercer día de ingreso hospitalario le colocan un Drum antecubital para garantizar una vía de acceso venoso en caso de hemorragia grave. En la madrugada siguiente, unas 20 horas después de la colocación del referido Drum, sufre un deterioro del estado general con sudoración profusa, vómitos y parada cardiorrespiratoria que, a pesar de las maniobras de reanimación, no se logra recuperar. Se le practica la autopsia médico-legal, tanto porque los médicos desconocen la causa del fallecimiento como porque los familiares, ante lo inesperado del fallecimiento, denuncian una supuesta mala praxis profesional.

En la autopsia se constata varón normoconstituido de 1,67 m, 80,5 kg e índice de masa corporal de 28,69 (sobrepeso), con tubo orotraqueal correctamente insertado y Drum antecubital en la flexura del codo derecho (Figura 1), bajo apósitos de gasa y vendas. Hemopericardio a tensión (Figura 2), se recogen $345 \mathrm{ml}$ de sangre líquida, diluida, con escasos coágulos. Evacuado el derrame pericárdico, se observan en la cavidad pericárdica $4 \mathrm{~cm}$ del extremo de un catéter venoso que sale por un orificio de unos 3 $\mathrm{mm}$ de diámetro en la cara anterior de la orejuela de la aurícula derecha (Figura 3). Los bordes de dicho orificio tienen un aspecto de desgarro irregular, lacerado, y tienen adherida fibrina y restos hemáticos coagulados. El catéter se continua con el Drum antecubital.

La cara interna de la orejuela derecha, en un área de $3,5 \times 2,5 \mathrm{~cm}$ de dimensiones máximas, que rodea al punto por el que se exterioriza el catéter, muestra en endocardio con numerosos puntos hemorrágicos y desprendimiento del endotelio (Figura 4).

El resto de los órganos no presentan alteraciones importantes, salvo intensa congestión generalizada.

El estudio histopatológico confirmó los hallazgos macroscópicos. En los análisis químico-toxicológicos sólo se detectaron los fármacos con los que estaba siendo tratado el paciente, en concentraciones terapéuticos.

\section{Discusión}

El taponamiento cardíaco es una complicación infrecuente, pero bien conocida, de los catéteres venosos centrales que a menudo es mortal. Generalmente se realiza una autopsia médico-legal.

Según las publicaciones y revisiones sobre el tema, la incidencia del taponamiento es muy variable y oscila entre el 0,0001\% y el 1,4\%. En todo caso, la 
mortalidad es muy alta, del $47 \%$ al $77 \%$, e incluso algunas publicaciones la elevan al $100 \%^{3}$.

En la mayoría de los hospitales, los catéteres insertados en venas periféricas, como el presente, pueden ser colocados por los profesionales de enfermería que están instruidos en la técnica, con el requisito de que inmediatamente después de la colocación se realice una radiografía para comprobar que la punta del catéter no ha llegado a las cavidades cardíacas.

Para evitar la complicación se recomienda una radiografía posteroanterior de tórax en la que se visualiza la posición de la punta del catéter. En general, se recomienda que el extremo del catéter no sobrepase más de $2 \mathrm{~cm}$ la línea que une la superficie inferior de las extremidades esternales de ambas clavículas (el llamado "criterio de Greenall") ${ }^{3}$. Otros autores, ante la imposibilidad de obtener en muchos pacientes, por su situación clínica, una radiografía en posición erecta, señalan que el catéter quede por encima de la unión entre el bronquio principal derecho y la tráquea ${ }^{3}$. En el año 1989, la Food and Drug Administration recomendó que el extremo distal del catéter no se colocara en las cavidades cardíacas, y que no se permita su migración a ellas, por el riesgo de graves complicaciones como arritmias o taponamiento cardíaco ${ }^{4}$.

El factor de riesgo fundamental para que ocurra esta complicación es la incorrecta posición de la punta del catéter, es decir, que esta quede en la aurícula o el ventrículo derechos y pueda perforar la pared de la cavidad. La perforación puede producirse tanto por los movimientos de la punta del catéter (por ejemplo, por los movimientos del brazo) como por los movimientos normales de las cavidades cardíacas, o por traumatismo directo durante la inserción.

En la actualidad, la mayoría de los catéteres centrales se insertan sobre guías más o menos flexibles, que navegan por el árbol vascular con facilidad y con menos posibilidad de traumatismo que el catéter (cuya punta es hueca). También existe la posibilidad de una perforación cardíaca o vascular por la guía, que es retirada al insertar el catéter, aun estando el catéter posicionado correctamente. En este caso sólo veríamos la perforación y sus consecuencias.

El punto de inserción del catéter es otro factor de riesgo, pues es mucho más frecuente el taponamiento en los insertados en venas periféricas, como el caso que nos ocupa, que en los insertados en venas centrales. Este riesgo puede atribuirse al movimiento de la punta del catéter con los cambios de posición del brazo, el cuello o la cabeza. Estudios en cadá-

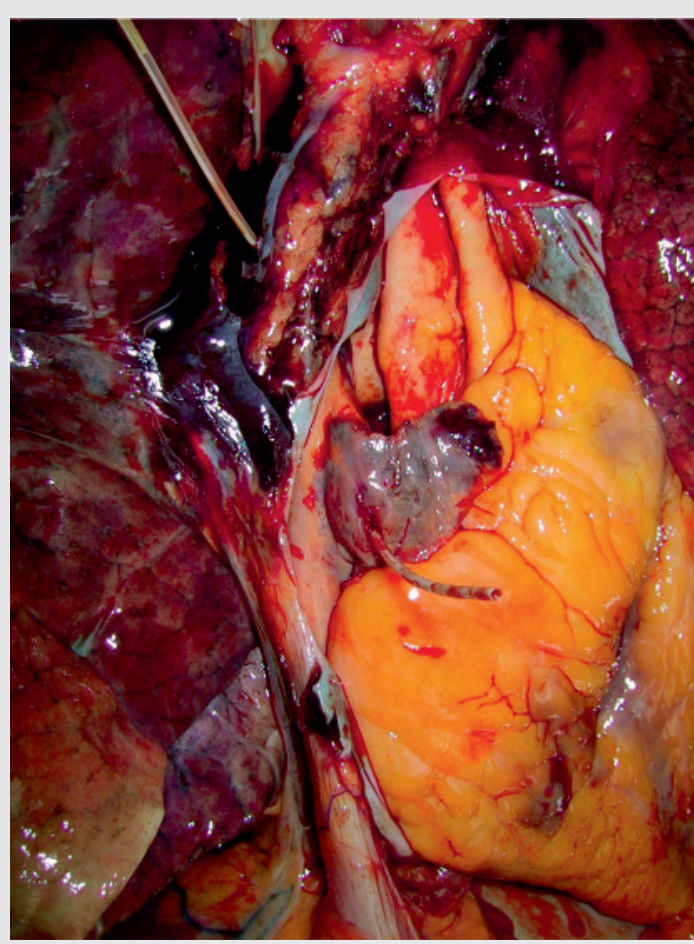

Figura 3.

Tras la evacuación del hemopericardio se objetiva el catéter que ha perforado la orejuela derecha.

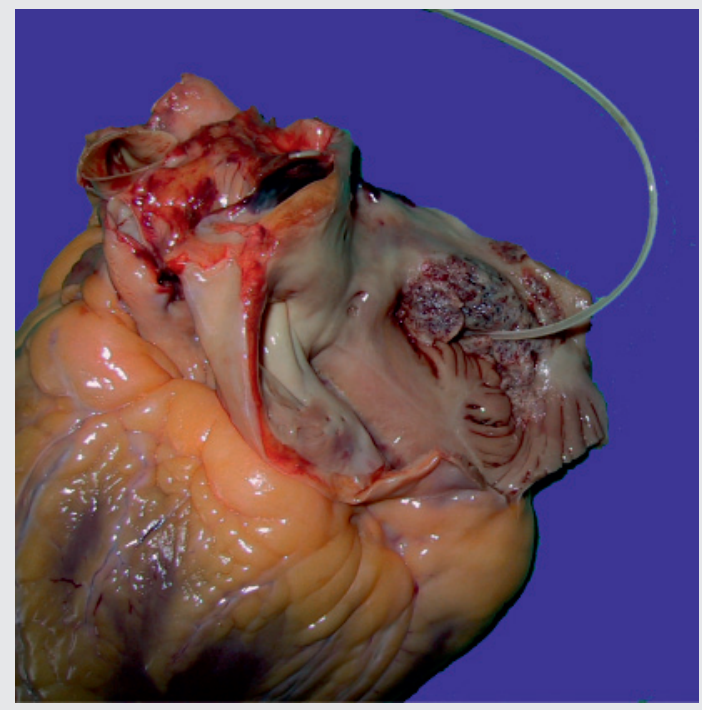

Figura 4.

Cara interna de la orejuela derecha en la que se evidencian las lesiones producidas por la punta del catéter antes de la perforación. veres han demostrado que los catéteres venosos centrales insertados en las venas antecubitales pueden avanzar hasta $7 \mathrm{~cm}$ con los movimientos de elevación y abducción del brazo. Otros estudios in vivo encuentran excesivo este dato y reducen el avance a sólo $2 \mathrm{~cm}^{5}$. 
Por último, la infusión de soluciones hiperosmolares, como ocurre en la nutrición parenteral, también es un factor de riesgo de taponamiento por catéter venoso central.

En el presente caso, nuestra opinión sobre el mecanismo de producción de la perforación es que la punta del catéter quedó en la orejuela derecha y, por la actividad cardíaca y los movimientos del brazo, se produjo la perforación.

La complicación puede presentarse en los minutos siguientes a la inserción del catéter o varios meses después. En el presente caso, los síntomas clínicos del taponamiento aparecieron a las 20 horas de su inserción y el fallecimiento a la media hora del comienzo de los síntomas. En el hospital no sospecharon que la causa de la muerte fuese el taponamiento, quizá por lo atípico de la sintomatología (intenso malestar general, sudoración profusa y vómitos). El taponamiento fue diagnosticado en la autopsia. Los síntomas suelen presentarse súbitamente y los más frecuentes y característicos son hipotensión, elevación de la presión venosa central y arritmias; sin embargo, en un tercio de los casos, la muerte, como ocurrió en este caso, ocurre de manera súbita y con vagos signos premonitorios ${ }^{3}$.
Alcanzar el volumen de derrame pericárdico que ocasiona la muerte por taponamiento cardíaco está en función de la velocidad con que se produzca su formación.

\section{Conclusiones}

El taponamiento cardíaco es una complicación infrecuente, pero extraordinariamente grave, de los catéteres venosos centrales. Cuando el paciente fallece, está indicada la autopsia médico-legal. Para su correcto reconocimiento en la autopsia, es muy importante que a los pacientes fallecidos en los hospitales y que han de ser sometidos a autopsia no se les retiren los dispositivos que tengan insertados (sondas de todo tipo, catéteres, drenajes, etc.), ni tampoco en la sala de autopsias hasta que se haya comprobado su correcta situación. Si el catéter hubiese sido retirado al comienzo de la autopsia o en el hospital, la tarea del patólogo forense de identificar el origen del taponamiento cardíaco y documentarlo adecuadamente habría sido más complicada.

Los autores declaran no tener ningún conflicto de intereses.

\section{Bibliografía}

1. Collier PE, Blocker SH, Graff DM, Doyle P. Cardiac tamponade from central venous catheters. Am J Surg. 1998;176(2):212-4.

2. Council of Europe. (2000). Recommendation $n^{\circ}$ R (99) 3 of the Committee of Ministers on the Harmonisation of Medico-Legal Autopsy. (Consultado el 6-11-2014.) Disponible en: http:// www.coe.int/t/dg3/healthbioethic/Texts_and_documents/default_en.asp. Traducción al español en Donat Laporta E. Rev Esp Med Leg. 1999; XXIII(86-87):90-103.
3. Booth SA, Norton B, Mulvey DA. Central venous catheterization and fatal cardiac tamponade. $\mathrm{Br} \mathrm{J}$ Anaesth. 2001;87(2):298-302.

4. US Food and Drug Administration. Precautions necessary with central venous catheters. FDA Drugs Bull. 1989;19:15-6.

5. Orme RM, McSwiney MM, Chamberlain-Webber RF. Fatal cardiac tamponade as a result of a peripherally inserted central venous catheter: a case report and review of the literature. $\mathrm{Br} J$ Anaesth. 2007;99(3):384-8. 